\title{
Ensaio clínico da griseofulvina e da terbinafina na terapia das dermatofitoses em cães e gatos
}

\author{
A clinical trial using griseofulvin and terbinafine in the treatment of canine and feline \\ dermatophytosis
}

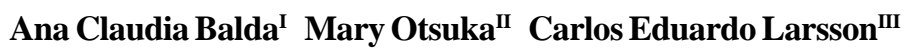

\section{RESUMO}

As dermatofitoses dos carnívoros domésticos são infecções fúngicas superficiais, causadas habitualmente por dois gêneros fúngicos: Microsporum sp e Trichophyton sp. Trata-se de uma antropozoonose, com importância na saúde pública. Objetivou-se comparar a eficácia da griseofulvina e da terbinafina na terapia das dermatofitoses. Trinta e cinco animais forma reunidos em três grupos de protocolos de terapia. A griseofulvina (50 mg/kg/dia) foi eficaz em 100\% dos casos, sem acarretar efeitos colaterais, com média de tempo para cura de 41 dias. Já a terbinafina na dose de cinco $\mathrm{mg} \mathrm{kg}^{-1}$ dia $^{-1}$ apresentou eficácia de $81,8 \%$, sem induzir efeitos colaterais e com êxito terapêutico em 21 dias. A dose de $20 \mathrm{mg} \mathrm{kg}^{-1} \mathrm{dia}^{-1}$ demonstrou a mesma eficácia que a dose de $5 \mathrm{mg} \mathrm{kg}^{-1} \mathrm{dia}^{-1}$, porém com efeitos colaterais observados em 16,6\% dos animais tratados, com tempo médio para cura de 33 dias. Conclui-se que a terbinafina é uma boa alternativa terapêutica, porém a griseofulvina ainda se constitui na droga de eleição para o tratamento das dermatofitoses de caninos e felinos.

Palavras-chave: dermatofitose, griseofulvina, terbinafina, cães, gatos.

\section{ABSTRACT}

Dermatophytoses in domestic carnivores are superficial infections caused mainly by two genera of fungi: Microsporum spp and Trichophyton spp. This disease is an anthropozoonosis important for public health. The goal of this study was to compare the efficacy of griseofulvin and terbinafine in the therapy of dermatophytosis. Thirty five animals were included in three protocols of treatment. Griseofulvin $(50 \mathrm{mg}$ $\mathrm{kg}^{-1}$ day $^{-1}$ ) was effective in $100 \%$ of the cases, with no side effects. The average time for cure was 41 days. Terbinafine used at a dosage of $5 \mathrm{mg} \mathrm{kg}^{-1}$ day-1 $^{-1}$ showed an efficacy of $81.3 \%$.
No side effects were observed and the average time for cure was 21 days. The same efficacy using a dosage of $20 \mathrm{mg} \mathrm{kg}^{-1}$ day $^{-1}$ of terbinafine was observed, although side effects were seen in $16.6 \%$ of the animals treated with an average time for cure of 33 days. The present study demonstrated that terbinafine is a good therapeutic alternative, although griseofulvin is still the first choice drug for the treatment of dermatophytosis in dogs and cats.

Key words: dermatophytosis, griseofulvin, terbinafine, dogs, cats.

\section{INTRODUÇÃO}

A dermatofitose é uma infecção fúngica superficial relativamente comum em carnívoros domésticos, causada por fungos queratinofílicos dos gêneros Microsporum spp e Trichophyton spp. O Microsporum canis (M. canis) é, sem dúvida, o dermatófito isolado com maior freqüência nas infecções dermatofíticas em diversas regiões do Brasil e do mundo, tanto na espécie felina, quanto na canina (GAMBALE et al., 1987; SPARKES et al., 1993; LARSSON et al., 1994; CABAÑES et al., 1997). A relevância dessa dermatopatia se dá não só pela freqüência com que é diagnosticada, mas principalmente por tratar-se de uma antropozoonose. Inúmeros pesquisadores referem-se à transmissão interespécie, em diferentes regiões, com percentís de ocorrência situados entre 18,7 e 87,0\% (WARNER, 1984;

ICentro Universitário das Faculdades Metropolitanas Unidas (UniFMU), Departamento de Clínica Médica da Faculdade de Medicina Veterinária e Zootecnia, Universidade de São Paulo (FMVZ, USP). Rua Carlos Weber, 633, apt. 134 A, 05303-000, São Paulo, SP, Brasil. E-mail: anabalda@terra.com.br. Autor para correspondência.

IIServiço de Dermatologia do Hospital Veterinário (HOVET), Departamento de Clínica Médica, FMVZ/USP, São Paulo, SP, Brasil.

IIIDepartamento de Clínica Médica, FMVZ/USP. São Paulo, SP, Brasil. 
PEPIN \& OXENHAM, 1986; CUADROS et al., 1990; PINHEIRO et al., 1997).

A griseofulvina, quando empregada na dose de 50-100 $\mathrm{mg} \mathrm{kg}^{-1}$ a cada 24 horas, por cerca de seis semanas, pode eventualmente acarretar efeitos colaterais (cutâneos, gastrointestinais, sangüíneos, hepáticos e neurológicos), mais observados em doses superiores a $100 \mathrm{mg} \mathrm{kg}^{-1}$ ou quando de uso prolongado, por mais de três meses (HELTON et al., 1986; KUNKLE \& MEYER, 1987). Há, porém, tendência de que a griseofulvina seja retirada do mercado, tal como já ocorreu na Bélgica (LATEUR, 2000).

A terbinafina é um antifúngico pertencente à classe das alilaminas, que possui atividade fungistática e fungicida contra dermatófitos e algumas leveduras, o que, na rotina clínica, redundaria em cura mais rápida e com redução nos índices de recidiva (VILLARS \& JONES, 1992). Há poucos dados disponíveis com relação aos efeitos colaterais e à eficácia clínica da terbinafina em animais. Estudos farmacocinéticos em cães e gatos sugeriam inicialmente doses em torno de $20 \mathrm{mg} \mathrm{kg}^{-1}$ a cada 24 ou 48 horas (Sparkes apud CARLOTTI, 1998; HNILICA, 1999; SCOTT et al. 2001). CHEN (2000) observou que, em doses de terbinafina variando entre 10 e $30 \mathrm{mg} \mathrm{kg}^{-1} \mathrm{dia}^{-1}$, a média de cura para os cães tratados foi de 7,6 semanas e para os gatos de 8,9 semanas, constatando um aumento das concentrações séricas das enzimas hepáticas, principalmente de ALT (alanina-aminotransferase) e FA (fosfatase alcalina), associado ao uso de doses mais elevadas da droga. CASTAÑON et al. (2001) trataram felinos portadores assintomáticos de M. canis com terbinafina na dose de $8,25 \mathrm{mg} \mathrm{kg}^{-1}$, obtendo cura micológica em três semanas de terapia. Existem alguns relatos humanos de fracasso na terapêutica de microsporíases tratadas com a terbinafina (PALACIO et al., 1998; CARAZO et al., 1999), principalmente nas dermatofitoses causadas pela espécie canis. KRAFCHIK \& PELLETIER (1999) sugeriram que talvez um aumento significativo na dose ou no tempo de terapia pudesse resultar em eficácia maior da droga nas infecções por $\boldsymbol{M}$. canis.
Os objetivos do presente trabalho foram os de avaliar a exeqüibilidade de adotar a terbinafina, como protocolo alternativo, na terapia das dermatofitoses de cães e gatos, considerando sua eficácia, o tempo de terapia necessário para a involução das lesões e para a negativação do exame micológico. Do mesmo modo, objetivou-se detectar eventuais efeitos colaterais decorrentes do emprego da droga e comparar tais características com aquelas da griseofulvina.

\section{MATERIAL E MÉTODOS}

Utilizaram-se 35 animais domiciliados (23 cães e 12 gatos) atendidos no Serviço de Dermatologia do Hospital Veterinário da FMVZ/USP. Vinte animais eram de raça definida, sendo que cinco destes eram felinos, todos da raça Persa; dentre os cães, três eram da raça Boxer, dois Yorkshire, dois Pinscher, dois Dachsund, um Bulldog inglês, um Lhasa Apso, um Shar-Pei e um Poodle. As idades variaram entre um e 108 meses, com média de 27,6 meses. Vinte e um animais eram machos, todos inteiros. A evolução do quadro de dermatofitose variava entre uma semana e 11 meses.

O diagnóstico foi estabelecido pelos aspectos clínicos, pelo exame através da luz de Wood e pela positividade da cultura fúngica em MycoselAgar e da análise da morfologia microscópica por microcultivo em lâmina com meio de Lactrimel a $25^{\circ} \mathrm{C}$. Os animais, após estabelecimento do diagnóstico, foram randomicamente reunidos em três grupos (Tabela 1). Os animais do Grupo I (nove cães e três gatos) foram submetidos à terapia com griseofulvina na dose de $50 \mathrm{mg} / \mathrm{kg}$, por via oral, a cada 24 horas. Os animais dos Grupos II (oito cães e três gatos) e III (seis cães e seis gatos) foram tratados, respectivamente, com cinco e $20 \mathrm{mg} \mathrm{kg}^{-1}$ de terbinafina, por via oral. Para os banhos rotineiros, recomendou-se que se utilizasse sabonete sem ação fungicida ou anti-séptica à base de cocoil isetionato de sódio, ácido esteárico, sabão à base de coco, óxido de zinco, dióxido de titânio, EDTA, EDTF $\left(\right.$ Dove $\left.^{\circledR}\right)$.

Os animais de todos os três grupos foram submetidos a controle clínico quinzenal indagando-

Tabela 1 - Características dos 35 animais (cães e gatos) com dermatofitose segundo o tipo de terapia griseofulvina (50mg kg-1) - Grupo I, terbinafina (5mg kg-1) - Grupo II e terbinafina $\left(20 \mathrm{mg} \mathrm{kg}^{-1}\right)-$ Grupo III.

\begin{tabular}{|c|c|c|c|c|c|}
\hline \multirow{2}{*}{ Grupo (nº de animais) } & \multicolumn{2}{|c|}{ Sexo } & \multirow{2}{*}{ Média de idade (meses) } & \multicolumn{2}{|c|}{ Microsporum } \\
\hline & M & $\mathrm{F}$ & & canis & gypseum \\
\hline I (12) & 8 & 4 & 25,4 & 10 & 2 \\
\hline II (11) & 6 & 5 & 49,4 & 9 & 2 \\
\hline III (12) & 7 & 5 & 9,9 & 12 & --- \\
\hline
\end{tabular}


se, nesse retorno, sobre a ocorrência de eventuais efeitos colaterais, examinando-se os animais, colhendose material para cultivo micológico e para avaliação da função hepática, pela determinação das concentrações séricas de alanina aminotransferase (ALT), aspartato aminotransferase (AST) e fosfatase alcalina (FA).

A suspensão da terapia e a preconização da alta foram embasadas na cura clínica, definida como total remissão da lesão com repilação, e na negativação de dois exames micológicos consecutivos com intervalo quinzenal executados a partir de pelame avulsionado da área lesionada e a partir de pelame colhido pelo Método do carpete.

\section{RESULTADOS}

Eficácia terapêutica da griseofulvina (50mg/kg) e da terbinafina (5 e $20 \mathrm{mg} \mathrm{kg}^{-1}$ )

A média temporal para a negativação do cultivo micológico dos animais tratados com griseofulvina (Grupo I) foi de 40,8 dias, com variação de 15 a 60 dias. Houve cura, clínica e micológica, em 100,0\% dos animais (Tabela 2). Todos os proprietários notaram melhora progressiva em seus animais. A média de tempo para negativação do cultivo micológico nos animais incluídos no grupo II (terbinafina na dose de $5 \mathrm{mg} \mathrm{kg}^{-1}$ ) foi de 21,6 dias; variando de 15 a 30 dias. Houve falha da terapia em dois animais (um cão e um gato); portanto, a eficácia da droga nesse grupo foi da ordem de 81,8\% (Tabela 2). Já com relação ao Grupo III, ou seja, aos animais que receberam tratamento a base de terbinafina na dose de $20 \mathrm{mg} \mathrm{kg}^{-1}$, a média de negativação do cultivo micológico foi de 33 dias, variando de 15 a 60 dias. Houve falha terapêutica, também, em dois animais (um cão e um gato); portanto, a eficácia da droga foi, igualmente, de 81,8\% (Tabela 2).

Análise estatística

Comparação entre eficácia e grupo

Para identificar possíveis associações entre

Tabela 2 - Griseofulvina e terbinafina na terapia da dermatofitose: negativação do cultivo micológico.

\begin{tabular}{cccc}
\hline \multicolumn{4}{c}{ Cultivo micológico negativo (n ${ }^{\circ}$ de animais) } \\
\hline Tempo & $\begin{array}{c}\text { Griseofulvina } \\
\left(50 \mathrm{mg} \mathrm{kg}^{-1}\right)\end{array}$ & $\begin{array}{c}\text { Terbinafina } \\
\left(5 \mathrm{mg} \mathrm{kg}^{-1}\right)\end{array}$ & $\begin{array}{c}\text { Terbinafina } \\
\left(20 \mathrm{mg} \mathrm{kg}^{-1}\right)\end{array}$ \\
\hline Dia 15 & $1(12)$ & $3(11)$ & $2\left(11^{*}\right)$ \\
Dia 30 & $4(12)$ & $9(11)$ & $6(11)$ \\
Dia 45 & $9(12)$ & $9(11)$ & $8(11)$ \\
Dia 60 & $12(12)$ & $9(11)$ & $9(11)$ \\
\hline
\end{tabular}

*A terapia foi suspensa em um dos animais em função de efeitos colaterais observados. eficácia e os grupos de animais com diferentes tratamentos, foi utilizado o teste Qui-Quadrado. Na tabela 3, encontram-se os percentuais encontrados para os cruzamentos e também os resultados dos testes, nos quais considerou-se haver diferença, estatisticamente significativa, quando $\mathrm{p}<0,05$. Podese observar, pelos resultados dispostos na tabela 3, que não há associação, ou seja, não há indicações estatisticamente significantes de que a eficácia foi maior em um dos grupos.

Comparação entre tempo de melhora e grupos

Para esta comparação, também utilizou-se o teste Qui-quadrado. Na tabela 3, estão dispostos os percentuais encontrados para os cruzamentos e também os resultados dos testes, onde não há indicações $(\mathrm{P}>0,05)$, estatisticamente significantes, de que as distribuições de períodos de melhora foram diferentes para algum dos grupos. Na tabela 4, encontram-se dispostos os dados relacionados à negativação do cultivo micológico.

Efeitos colaterais decorrentes dos protocolos de terapia à base de griseofulvina ( $\left.50 \mathrm{mg} \mathrm{kg}^{-1}\right)$ e de terbinafina (5 e $20 \mathrm{mg} \mathrm{kg}^{-1}$ )

Com relação aos eventuais efeitos colaterais decorrentes do emprego da griseofulvina na dose de $50 \mathrm{mg} \mathrm{kg}^{-1}$ e da terbinafina na dose de $5 \mathrm{mg} \mathrm{kg}^{-1}$, não foi observada qualquer manifestação sintomática que pudesse ser atribuída aos fármacos. Quanto aos resultados das dosagens enzimáticas de ALT, AST e FA, não foram observadas alterações dignas de nota, em nenhum dos animais tratados. Houve, ainda, uma boa aceitação por parte dos proprietários com relação ao custo da prescrição.

Com relação aos animais tratados com terbinafina na dose de $20 \mathrm{mg} \mathrm{kg}^{-1}$, observou-se quadro gastroentérico em dois (16,6\%) dos 12 animais (um cão e um gato) incluídos no Grupo III. Além disso, um animal da espécie felina, além de quadro emético, apresentou aumento transitório da atividade sérica de ALT e FA, sendo que, no trigésimo dia de terapia, e após a suspensão da droga, houve normalização da função hepática com remissão do quadro clínico. O cão apresentou diarréia aquosa, anorexia e vários episódios eméticos sendo que, no sétimo dia de terapia, houve remissão do quadro após a suspensão da terbinafina. Com relação ao custo, houve um incremento importante nesse grupo e conseqüentemente uma aceitação menor pelos proprietários. 
Tabela 3 - Eficácia das drogas para os animais incluídos nos grupos I, II e III.

\begin{tabular}{|c|c|c|c|c|c|c|c|c|}
\hline & & \multicolumn{6}{|c|}{ Grupos } & \multirow{4}{*}{ p-valor } \\
\hline & & \multicolumn{2}{|c|}{ I } & \multicolumn{2}{|c|}{ II } & \multicolumn{2}{|c|}{ III } & \\
\hline & & \multicolumn{2}{|c|}{$\begin{array}{l}\text { Griseofulvina } \\
\left(50 \mathrm{mg} \mathrm{kg}^{-1}\right)\end{array}$} & \multicolumn{2}{|c|}{$\begin{array}{l}\text { Terbinafina } \\
\left(5 \mathrm{mg} \mathrm{kg}^{-1}\right)\end{array}$} & \multicolumn{2}{|c|}{$\begin{array}{l}\text { Terbinafina } \\
\left(20 \mathrm{mg} \mathrm{kg}^{-1}\right)\end{array}$} & \\
\hline & & $\mathrm{N}$ & $\%$ & $\mathrm{~N}$ & $\%$ & $\mathrm{~N}$ & $\%$ & \\
\hline \multirow{2}{*}{ Eficácia } & Não & & & 2 & 18,2 & 2 & 18,2 & \multirow{2}{*}{0.290} \\
\hline & Sim & 12 & 100 & 9 & 81,8 & 9 & 81,8 & \\
\hline
\end{tabular}

\section{DISCUSSÃo}

Os resultados obtidos através da terapia à base de griseofulvina na dose de $50 \mathrm{mg} \mathrm{kg}^{-1}$, a cada 24 horas, durante uma média de 41 dias, permitiram considerar a griseofulvina como a droga de eleição para o tratamento de dermatofitose em cães e gatos, fato esse que havia sido ressaltado por outros autores (JAHAM et al., 2000).

Com relação ao grupo II, tratado com terbinafina na dose de $5 \mathrm{mg} \mathrm{kg}^{-1}$ de peso, obteve-se uma eficácia de $81,8 \%$, valor este inferior àquele obtido pela terapia à base de griseofulvina, porém com um tempo médio para cura de 22 dias, fato esse já esperado, levando-se em conta a atividade fungicida da terbinafina. Neste grupo de animais, não foram observados efeitos colaterais farmacodérmicos e/ou sistêmicos, tampouco aumento nas concentrações das atividades enzimáticas. O custo da medicação foi considerado viável pelos proprietários. Apesar de não haver diferença estatisticamente significativa nem com relação à eficácia e nem ao tempo de melhora entre os três Grupos, deve-se ressaltar que há uma limitação do estudo com relação ao número de animais incluídos, o que ocorreu provavelmente em função do número de retornos aprazados para os animais e conseqüente perda de quase metade dos casos inicialmente acompanhados.

Com a dose de $20 \mathrm{mg} \mathrm{kg}^{-1}$, a cada 24 horas, administrada aos 12 animais do Grupo III, obteve-se eficácia da ordem de $81,8 \%$, idêntica àquela obtida com a dose de $5 \mathrm{mg} \mathrm{kg}{ }^{-1}$. De forma insólita, o tempo médio de cura foi de 33 dias, com extremos entre $15 \mathrm{e}$ 60 dias. Este período mais longo pode ser provavelmente explicado pela inclusão, no grupo III, de dois animais com quadro clínico generalizado, como os demais, embora bem mais grave. Além disso, é provável que haja uma relação entre o tempo de terapia necessário para cura e o agente etiológico da dermatofitose, já que o grupo III foi constituído em sua totalidade por animais com dermatofitose causada por $\boldsymbol{M}$. canis. Na espécie humana, observou-se a necessidade de prolongamento da terapia por três a quatro semanas em microsporíases causadas pela espécie canis (KRAFCHIK \& PELLETIER, 1999).

O período médio de 21 e 33 dias, respectivamente para os grupos I e II, para a melhora clínica e a negativação do exame micológico, difere daquele observado por CHEN (2000), que foi de 7,6 semanas para cães e 8,9 semanas para gatos, em animais tratados com terbinafina em doses variando de 10 a $30 \mathrm{mg} \mathrm{kg}^{-1} \mathrm{dia}^{-1}$.

Houve manifestação de quadro gastroentérico em um cão e aumento da atividade das enzimas hepáticas em um dos gatos, acompanhados de quadro emético, fatos estes transitórios, já que houve remissão após a suspensão do tratamento, levando a crer-se que tais manifestações clínicas ocorreram em função da administração da terbinafina. Aumento semelhante das concentrações séricas de enzimas hepáticas já havia sido constatado por CHEN (2000), quando da utilização da dose de $30 \mathrm{mg} \mathrm{kg}^{-1}$.

A terbinafina talvez não substitua a griseofulvina, em função do custo, porém, na dose de $5 \mathrm{mg} \mathrm{kg}{ }^{-1}$, poderá ser uma excelente alternativa de tratamento em médio prazo, mormente se comercializada sob a forma de medicamento genérico.

\section{CONCLUSÕES}

Os resultados obtidos no presente estudo permitiram concluir que a terbinafina é uma droga eficaz e relativamente segura no tratamento das dermatofitose em cães e gatos, principalmente quando utilizada na dose de $5 \mathrm{mg} \mathrm{kg}^{-1}$, dose que também torna a terbinafina

Tabela 4 - Tempo médio para a cura (em dias) dos animais incluídos nos grupos I, II e III.

\begin{tabular}{|c|c|c|c|c|}
\hline & \multicolumn{3}{|c|}{ Grupo } & \multirow{3}{*}{ P-valor } \\
\hline & I & II & III & \\
\hline & Griseofulvina (50mg kg ${ }^{-1}$ ) & Terbinafina $\left(5 \mathrm{mg} \mathrm{kg}^{-1}\right)$ & Terbinafina (20mg kg-1) & \\
\hline Tempo médio para cura (dias) & \pm 14 & \pm 25 & \pm 14 & $\mathrm{P}>0,3$ \\
\hline
\end{tabular}

Ciência Rural, v.37, n.3, mai-jun, 2007. 
economicamente viável aos proprietários. Porém, se confirmou-se novamente que a griseofulvina na dose de $50 \mathrm{mg} \mathrm{kg}^{-1}$ ainda permanece como droga de escolha nas infecções dermatofíticas por se mostrar uma droga bastante efetiva, desprovida de efeitos colaterais e de custo bastante acessível aos proprietários.

\section{REFERÊNCIAS}

CABAÑES, F.J et al. Dermatophytosis isolated from domestic animals in Barcelona, Spain. Mycopathologia, v.137, n.2, p.107-113, 1997.

CARAZO, J.L.S. et al. Tratamiento actual de las micosis superficiales. Revista Iberoamericana de Micologia, v.16, n.26, p.26-30, 1999.

CARLOTTI, D.N. Traitement des teignes chez le chat. Le Point Vétérinaire, v.29, n.193, p.681-689, 1998.

CASTAÑON-OLIVARES, L.R. et al. Effectiveness of terbinafine in the eradication of Microsporum canis from laboratory cats. Mycoses, v.44, n.3-4, p.95-97, 2001.

CHEN, C. The use of terbinafine for the treatment of dermatophytosis. Veterinary Dermatology, San Francisco, v.11, n.1, p.41, 2000. (Scientific abastracts - Fourth World Congress of Veterinary Dermatology).

CUADROS, J.A. et al. Dermatophytosis in a urban setting: prospective study of 135 cases. Enferm Infectious Microbiology Clinics, v.8, n.7, p.429-433, 1990.

GAMBALE, W. et al. Incidência de micoses superficiais em São Paulo, Capital. Anais Brasileiros de Dermatologia, v.62, n.4, p.193-194, 1987.

HELTON, K.A. et al. Griseofulvin toxicity in cats. Literature review and report of seven cases. Journal of the American Hospital Association, v.22, p.453-458, 1986.

HNILICA, K.A. Dermatophytosis. In: ANNUAL MEMBER'S MEETING, 1999, Maui, Hawaii. Proceedings... Maui, Hawaii: American Academy of Veterinary Dermatology, 1999. p.8-9.
JAHAM, C. et al. Tradicional antifungal dermatologic agents. Compendium of Continuing Education, v.22, n.5, p.461468, 2000.

KUNKLE, G.A.; MEYER, D.J. Toxicity of high doses of griseofulvin in cats. Journal of the American Veterinary Medical Association, v.191, n.3, p.322-323, 1987.

KRAFCHIK, B.; PELLETIER, J. An open study of tinea capitis in 50 children treated with a 2 week course of oral terbinafine. Journal of the American Academy of Dermatology, v.41, n.1, p.60-63, 1999.

LARSSON, C.E. et al. Ringworm in domestic cats in São Paulo, Brazil between 1981-1990. Feline Practice, v.22, n.3, p.219222, 1994.

LATEUR, N. Dermatophytosis due to domestic animals. Reviste Medicalle des Bruxeles, v.21, n.4, p.237-241, 2000.

PALACIO, A. et al. Terbinafine (Lamisil) therapy for Microsporum canis scalp ringworm. Clinical and Experimental Dermatology, v.23, p.142-143, 1998.

PEPIN, G.A.; OXENHAM, M. Zoonotic dermatophytosis (ringworm). Veterinary Record, v.118, p.110-111, 1986.

PINHEIRO, A.Q. et al. Dermatophytosis in the urban environment and the coexistence of man with dogs and cats. Revista da Sociedade Brasileira de Medicina Tropical, v.30, n.4, p.287-294, 1997.

SCOTT, D.W. et al. Fungal skin diseases. In: Small animal dermatology. 6.ed. Philadelphia: Saunders, 2001. p.336-422.

SPARKES, A.H. et al. Epidemiological and diagnostic features of canine and feline dermatophytosis in the United Kingdom from 1956 to 1991 . Veterinary Record, v.133, n.3, p.5761, 1993.

VILLARS, V.V.; JONES, T.C. Special features of the clinical use of oral terbinafine in the treatment of fungal diseases. British Journal of Dermatology, v.126, n.39, p.61-69, 1992.

WARNER, R.D. Ocurrence and impact of zoonoses in pet dogs and cats at USA Air Forces bases. American Journal of Public Health, v.74, p.1239-1242, 1984. 